\title{
Prevention of catheter-related infection: which catheter, which access and which insertion technique should be chosen?*
}

\author{
Prévention des infections liées aux cathéters : quel cathéter, quelle voie et quelle technique \\ d'insertion choisir?
}

\section{Lorente}

Revised: 8 October 2012, Accepted: 6 November 2012

(C) SRLF et Springer-Verlag France 2012

\begin{abstract}
Central venous and arterial catheters are commonly used in critically ill patients. Such catheters may entail mechanical and infectious complications. Catheter-related infections result in a high rate of morbidity and mortality and elevated costs. Numerous contributions have been made in the prevention of catheter-related infections, and the present review focuses on which catheter, which access and which insertion technique should be used. Regarding vascular access, some sites have shown higher risk of catheter-related bloodstream infections (CRBSI), such as the internal jugular site with tracheostomy and the femoral access site. With respect to which catheter should be used, there is evidence that catheters impregnated with rifampicin-(minocycline or miconazole) and chlorhexidine-silver sulfadiazine reduce the risk of CRBSI. These impregnated catheters could be considered in the following circumstances: vascular access channelling with increased risk of CRBSI (such as the internal jugular with tracheostomy or femoral access), immunocompromised patients or patients with disorders of skin integrity. Regarding the choice of insertion technique, there is evidence that ultrasound guidance may decrease cannulation failure and complication rates.
\end{abstract}

Keywords Catheter · Venous - Arterial - Prevention · Impregnated · Ultrasounds · Bloodstream · Femoral · Subclavian · Jugular $\cdot$ Radial $\cdot$ Cubital $\cdot$ Brachial $\cdot$ Dorsalis pedis

\section{Lorente $(\square)$}

Intensive Care Unit, Hospital Universitario de Canarias, Ofra, s/n. La Laguna-38320, Santa Cruz de Tenerife, Spain e-mail : lorentemartin@msn.com

\footnotetext{
* Cet article correspond à la conférence faite par l'auteur au congrès de la SRLF 2013 dans la session : Catheter infection: what's new?
}

Résumé Les cathéters veineux centraux et artériels sont souvent utilisés chez les patients de réanimation. Ces cathéters peuvent engendrer des complications infectieuses et mécaniques. L'infection liée au cathéter est associée à une morbimortalité élevée et à un surcoût important. Cette revue générale s'intéresse à l'impact du type de cathéter, du site d'insertion et de la technique de cathétérisme sur la survenue d'infection liée au cathéter. Concernant le site d'insertion du cathéter, certains sites ont été identifiés comme facteurs de risque de bactériémie liée au cathéter, tels que le site jugulaire en présence d'une canule de trachéotomie et le site fémoral. Le choix du type de cathéter influence également l'incidence de colonisation et d'infection liées au cathéter. Les cathéters imprégnés d'agents anti-infectieux (minocycline-rifampicine ou rifampicine-miconazole) ou antiseptiques (chlorhexidine-sulfadiazine argentique) sont associés à une réduction de l'incidence d'infection liée au cathéter. L'utilisation de ces cathéters pourrait être proposée dans les circonstances suivantes : cathéter tunnelisé chez un patient à risque d'infection liée au cathéter (par exemple accès jugulaire interne avec trachéotomie ou accès fémoral), patients immunodéprimés ou souffrant de lésions cutanées. L'utilisation de l'échographie permet de réduire le taux d'échec et les complications mécaniques.

Mots clés Cathéter · Veineux · Artériel · Prévention Imprégnés · Bactériémie $\cdot$ Fémoral $\cdot$ Sous-clavier . Jugulaire $\cdot$ Radial $\cdot$ Cubital $\cdot$ Brachial $\cdot$ Dorsal $\cdot$ Pédieux

\section{Introduction}

Central venous and arterial catheters are commonly used in critically ill patients [1]. Such catheters may entail mechanical and infectious complications. The interest in catheterrelated infection lies in attributable morbidity and mortality and the costs involved [2]. Numerous contributions have 
been made in the field to prevent catheter-related infections, and the present review focuses on certain aspects of prevention, namely which catheter, which access and which insertion technique should be used.

\section{Which central venous catheter access}

\section{Different central venous catheter accesses}

A strategy that may help to reduce the incidence of catheterrelated infection is based on the choice of venous access. Some studies have found higher catheter tip colonization in femoral than in jugular and subclavian accesses [3-5], in femoral than in subclavian accesses [6,7] and in jugular than in subclavian accesses $[7,8]$.

In relation to the incidence of catheter-related bloodstream infections (CRBSI) associated with different central venous catheter (CVC) accesses, in a study carried out by our team including 2595 venous catheters (917 subclavian, 1390 jugular and 288 femoral catheters), we found a higher CRBSI incidence density for femoral than for jugular ( 8.34 vs. 2.99 events of CRBSI/1000 catheter days, $p=0.002$ ) and subclavian accesses ( 8.34 vs. 0.97 events of CRBSI/1000 catheter days, $p<0.001)$ and higher for jugular than for subclavian access (2.99 vs. 0.97 events of CRBSI/1000 catheter days, $p=0.005)$ [9].

A systematic review recently published by Marik et al. [10], including two randomized controlled trials (RCT) and eight cohort studies, found no significant difference in the risk of CRBSI between the femoral and subclavian sites, and between the femoral and internal jugular sites. However, it is not clear what criteria motivated the decision to exclude from the analysis two studies comparing femoral and internal jugular sites. Including these two studies, the risk of CRBSI was higher in femoral than in internal jugular sites (risk ratio $=1.90 ; 95 \%$ confidence interval $(\mathrm{CI})=1.21-2.97$; $p=0.005)$ [11].

The same systematic review [10] found no significant difference in the risk of deep venous thrombosis between the femoral and subclavian/jugular sites. However, some explanations about the two RCTs included in the analysis are necessary. In the RCT published by Merrer et al. [6], 289 patients were randomly assigned to central venous catheterization at either the femoral site $(n=145)$ or the subclavian site $(n=144)$. Femoral catheterization was associated with a higher incidence of overall thrombotic complications ( 21.5 vs. $1.9 \% ; p<0.001)$ and complete thrombosis of the vessel ( 6 vs. $0 \% ; p=0.01)$. Femoral catheterization was the only risk factor for thrombotic complications (odds ratio $=$ 14.42 ; $95 \% \mathrm{CI}=3.33-62.57 ; p<0.001)$. In the RCT conducted by Parienti et al. [12], 750 patients were assigned to central venous catheterization at either the femoral site $(n=370)$ or the jugular site $(n=366)$; the analysis of deep venous thrombosis showed no significant differences between the femoral and jugular groups (8/76 (10.5\%) vs. $17 / 75(22.7 \%) ; p=0.16)$.

\section{Different internal jugular venous accesses}

Another aspect in relation to CRBSI, according to CVC site, is the influence of the different internal jugular venous accesses, which has scarcely been studied. Our team conducted a study comparing 515 internal jugular venous catheters by central access and 169 by posterior access; we found a higher incidence of CRBSI in the central access group than in the posterior access group ( 4.8 vs. 1.2 events of CRBSI/1000 catheter days; odds ratio $=3.9 ; 95 \% \mathrm{CI}=1.1$-infinite; $p=0.03$ ) [13]. This was probably due to the lower contamination by oropharyngeal secretions in the posterior access group. As the patients are placed in a semirecumbent position (by elevating the head of the bed) and the puncture site for posterior access is higher than that used for central access, oropharyngeal secretions reach the central access more easily than the posterior access due to gravity. The semirecumbent position is used to decrease the risk of oesophageal reflux and subsequent aspiration, as recommended in the guidelines for the prevention of ventilator-associated pneumonia of the Society for Healthcare Epidemiology of America/Infectious Diseases Society of America (SHEA/IDSA) [14].

After the study demonstrated the influence of the jugular access in the incidence of CRBSI [13], we carried out other analyses comparing CRBSI rates between femoral and central internal jugular accesses [15], and between posterior internal jugular and subclavian venous accesses [16].

As there was a higher incidence of CRBSI in femoral than in jugular sites $[9,10]$, higher in central than in posterior jugular access [13] and in all the studies included in the systematic review by Marik et al. [10], the internal jugular access used (posterior, central or anterior) was not specified; we believed it could be interesting to compare the incidence of CRBSI between the femoral and central jugular accesses. We carried out a study including 208 femoral catheters and 515 central internal jugular venous catheters and found a higher incidence of CRBSI in femoral than in central internal jugular venous access (9.52 vs. 4.83 events of CRBSI/1000 catheter days; risk ratio $=1.93 ; 95 \% \mathrm{CI}=1.03-3.73$; $p=0.04)[15]$.

Similarly, as there was a higher incidence of CRBSI associated with jugular than with subclavian sites [9] and the relevant studies included in the systematic review by Marik et al. [10] did not specify which internal jugular access was used, we aimed to compare CRBSI rates between the subclavian and posterior jugular accesses. We carried out an analysis of 877 subclavian and 169 posterior internal jugular venous accesses and found no differences in the incidence 
of CRBSI (1.02 vs. 1.21 events of CRBSI/1000 catheter days; $p=0.99)[16]$.

\section{Tracheostomy}

There are scarce data about the influence of tracheostomy on the incidence of catheter-related infection [17-20]. In a study by Michel et al. [17] with 390 subclavian catheters, patients with tracheostomy had a higher incidence of catheter-tip colonization compared with those without tracheostomy (34 vs. $7 \%$ ). In a study by Dusan et al. [18] with 219 catheters sited in subclavian or jugular accesses, patients with tracheostomy also had a higher incidence of catheter-tip colonization compared with those without tracheostomy (24 vs. $13 \%$ ). In a study by Garnacho-Montero et al. [19] with 1211 subclavian or jugular catheters, tracheostomy was found to be an independent risk factor for CRBSI. In another study by our team, we analyzed a total of 515 internal jugular venous catheters (52 with tracheostomy and 463 without tracheostomy) and 877 subclavian venous catheters ( 89 with tracheostomy and 788 without tracheostomy). We found a higher incidence of CRBSI in patients with tracheostomy than in those without tracheostomy (11.25 vs. 1.43 events of CRBSI/1000 catheter days; odds ratio $=7.99 ; 95 \% \mathrm{CI}=4.38$-infinite; $p<0.001$ ) and a higher incidence of CRBSI in patients with tracheostomy using the jugular access than with subclavian access (21.64 vs. 5.11 events of CRBSI/1000 catheter days; odds ratio $=4.23 ; 95 \% \mathrm{CI}=1.44$-infinite; $p=0.01$ ) [20].

After the finding of the influence of tracheostomy on the incidence of CRBSI at subclavian and jugular accesses, we believed it could be interesting to compare the incidence of CRBSI between central internal jugular venous catheters in the presence of tracheostomy and femoral venous catheters. We included 52 central internal jugular catheters with tracheostomy and 208 femoral catheters and found a higher incidence of CRBSI in patients with central internal jugular catheters than in those with femoral site catheters (21.64 vs. 9.52 events of CRBSI $/ 1000$ catheter days; risk ratio $=2.27$; $95 \% \mathrm{CI}=1.04-4.97 ; p=0.04)[21]$.

We also carried out another analysis to compare the incidence of CRBSI between the subclavian venous catheter site in the presence of tracheostomy $(n=147)$ and the femoral venous catheter site $(n=313)$. We found a lower incidence of CRBSI in subclavian venous catheters in the presence of tracheostomy than in femoral venous catheters (3.9 vs. 10.1 events of CRBSI $/ 1000$ catheter days; odds ratio $=0.39 ; 95 \%$ $\mathrm{CI}=0.001-0.910 ; p=0.03)$ [22]

Finally, we also performed another analysis to determine the influence of the presence of tracheostomy on CRBSI rates in patients with posterior jugular catheters. We included 16 catheterizations with tracheostomy and 153 catheterizations without tracheostomy and found a higher incidence of CRBSI in posterior jugular access in patients with tracheos- tomy than in those without tracheostomy (13.24 vs. 0 events of CRBSI $/ 1000$ catheter days; odds ratio $=23.92 ; 95 \% \mathrm{CI}=$ 1.86-infinite; $p=0.008$ ) [23].

The Centers for Disease Control (CDC) and IDSA guidelines for the prevention of intravascular catheter-related infections recommend [24]: the avoidance of the femoral vein (Category IA) and the use of the subclavian site rather than jugular or femoral sites to minimize the risk of infection for non-tunneled CVC placement (Category IB). There is no recommendation about the presence of tracheostomy and the different jugular venous access sites.

On the basis of published findings, I suggest the following central venous catheter sites to minimize the risk of CRBSI: internal jugular by posterior access without tracheostomy or subclavian without tracheostomy, internal jugular by central access without tracheostomy, subclavian with tracheostomy, femoral, internal jugular by posterior access with tracheostomy and finally internal jugular by central access with tracheostomy.

\section{Which arterial catheter access}

There are scarce data about arterial catheter-related infection according to the different sites. In a review by Scheer et al. [25], the three most commonly used arterial cannulation sites were closely examined. The review included a total of 19,617 radial, 3899 femoral and 1989 axillary artery catheterizations. Major complications (such as permanent ischaemic damage, sepsis and pseudoaneurysm formation) occurred in less than $1 \%$ of the cases, and rates were similar for the radial, femoral and axillary arteries. The authors commented that other arteries employed for catheterization, such as the brachial, dorsal pedis, ulnar, tibial and temporal arteries, have been used without serious complications, but data were not reported. The authors opted not to perform statistical analyses because the studies selected in the review did not report data on all the different arterial catheter sites.

In some studies comparing different arterial accesses, no significant differences in the incidence of arterial catheterrelated infection were found [26-31]. One study found a higher incidence of arterial catheter colonization in femoral than in radial and cubital sites [32]. An important limitation of these studies is the small sample size.

Our team conducted a study including 2949 arterial catheters (2088 radial, 112 brachial, 131 dorsalis pedis and 618 femoral catheters) [33]. We found a higher incidence of arterial catheter-related infection in femoral than in radial access $(1.92$ vs. 0.25 events $/ 1000$ catheter days; odds ratio $=1.9$, $95 \% \mathrm{CI}=1.15-3.41 ; p=0.009)$ and no other statistically significant differences. After this study, we increased the number of catheters and found a higher incidence of arterial catheter-related infection in 1085 femoral than in 174 dorsalis 
pedis accesses $(p=0.01)$ [34], 141 brachial accesses $(p=0.02)$ [35] and 449 cubital accesses $(p=0.02)$ [36].

The CDC and IDSA guidelines for the prevention of intravascular catheter-related infections published in 2011 recommended that the use of the radial, brachial or dorsalis pedis sites is preferred over the femoral or axillary sites of insertion to reduce the risk of infection in adults (Category IB) [24]. I agree with the recommendation on avoiding the arterial femoral access.

\section{Which catheter}

A proposed strategy to reduce the incidence of CRBSI is the use of CVCs impregnated with different antimicrobial agents, such as chlorhexidine-silver sulfadiazine (CHSS), minocycline-rifampicin, cefazolin, vancomycin, heparin, chlorhexidine, silver, platinum and carbon.

A meta-analysis by Veenstra et al. [37], which included 11 RCT and 2603 catheters, found a lower incidence of CRBSI with the use of first-generation (only the external surface was impregnated) CHSS-impregnated catheters compared with non-impregnated catheters (odds ratio $=$ $0.56 ; 95 \% \mathrm{CI}=0.37-0.84 ; p=0.005)$. However, some RCTs performed after this meta-analysis reported that second-generation (both external and internal surfaces impregnated) CHSS-impregnated catheters showed a lower incidence of catheter-tip colonization than non-impregnated catheters, but there were no significant differences in the incidence of CRBSI [38-40].

Another meta-analysis by Falagas et al. [41], which included 3452 CVCs from 8 RCTs, showed decreased CRBSI with the use of rifampicin-impregnated catheters (rifampicin-minocycline in seven RCTs and rifampicinmiconazole (RM) in one RCT) compared with non-coated catheters.

Our team performed a study to determine the efficacy of RM-impregnated catheters to decrease the incidence of CRBSI [42]. We analyzed 184 femoral (73 RM and 111 standard catheters) and 241 central jugular venous catheters (114 RM and 127 standard catheters). We found a lower incidence of CRBSI with RM-impregnated catheters than with standard catheters in femoral access ( 0 vs. 8.62 events of CRBSI $/ 1000$ catheter days, odds ratio $=0.13,95 \%$ $\mathrm{CI}=0.00-0.86 ; p=0.03)$ and in central internal jugular access ( 0 vs. 4.93 events of CRBSI/1000 catheter days, odds ratio $=0.13,95 \% \mathrm{CI}=0.00-0.93 ; p=0.04)$.

A multicentre RCT by Darouiche et al. [43] found that the use of minocycline-rifampin-impregnated catheters $(n=356)$ was associated with a lower rate of CRBSI than CHSS-impregnated catheters $(n=382)(0.3$ vs. $3.4 \%$; $p<0.002)$.
A review by Ramritu et al. [44] found that first-generation CHSS and minocycline-rifampicin-impregnated catheters reduced the risk of CRBSI; however, second-generation CHSS-impregnated catheters, cefazolin, vancomycin, heparin, chlorhexidine, silver, platinum and carbon-impregnated catheters have not been found to reduce the risk of CRBSI.

The use of antimicrobial or antibiotic-impregnated catheters has been found to decrease the incidence of CRBSI and catheter-related cost in some cost-effectiveness analyses [45-47]. The mean additional cost due to CRBSI in the studies included in these cost-effectiveness analyses was approximately $\$ 10,000$; and in some studies this was as high as $\$ 40,000$ [48] and $\$ 71,000$ [49] due mainly to increased hospital stay of 24 and 22 days respectively. In these costeffectiveness studies, the catheter-related cost included the increase of hospital stay and this varied greatly between the different studies. However, there were no studies reporting catheter-related cost excluding the cost due to increased hospital stay. Thus, the objective of two studies carried out by our team was to determine the immediate catheter-related cost (including only the cost of CVC, diagnosis of CRBSI and antimicrobials for the treatment of CRBSI) using RM or standard catheters in femoral access [50] and in jugular venous access with tracheostomy [51]. In the femoral venous access analysis, which included 184 RM and 190 standard catheters, we found a higher incidence of CRBSI with standard than with RM catheters (8.61 vs. 0 CRBSI episodes/1000 catheter-days; odds ratio $=19.26 ; 95 \% \mathrm{CI}=3.24$-infinite; $p<0.001)$ and a higher immediate catheter-related cost per day with standard catheters than with RM catheters $(€ 18.22 \pm$ 53.13 vs. $12.61 \pm 8.38 ; p<0.001$ ) [50]. In the analysis of the jugular venous access with tracheostomy, which included 68 RM and 79 standard catheters, we found a lower incidence of CRBSI with RM than with standard catheters (0 vs. 20.16 CRBSI episodes $/ 1000$ catheter-days; odds ratio $=0.05 ; 95 \%$ $\mathrm{CI}=0.001-0.32 ; p<0.001)$ and a lower immediate catheterrelated cost per day with RM than with standard catheters $(€ 11.46 \pm 6.25$ vs. $38.11 \pm 77.25 ; p<0.001)$ [51].

The CDC and IDSA guidelines for the prevention of intravascular catheter-related infections published in 2011 recommended the use of a CHSS or minocycline-rifampicinimpregnated catheter in patients whose catheter is expected to remain in place $>5$ days and if the CRBSI rate has not decreased after the implementation of a comprehensive strategy to reduce it (Category IA); and the preventive strategy should include at least the following three components: educating persons who insert and maintain catheters, use of maximal sterile barrier precautions and a $>0.5 \%$ chlorhexidine preparation with alcohol for skin antisepsis during catheter insertion [24]. I have some comments about the CDC and IDSA recommendations regarding this issue. First, the guidelines recommended CHSS-impregnated catheters without specifying which generation of CHSS-impregnated catheter 
is recommended. This recommendation is based on RCTs showing that second-generation CHSS-impregnated catheters reduced the incidence of catheter-tip colonization [38-40]. In these RCTs there were no significant differences in the incidence of CRBSI with the use of second-generation CHSSimpregnated catheters; however, in all of them there was a tendency to lower CRBSI with antimicrobial catheters, and there is no published meta-analysis including these studies. On the other hand, there is evidence that first-generation CHSS-impregnated catheters reduced CRBSI according to the results of one meta-analysis [37]. Second, the CDC and IDSA guidelines recommended the use of minocyclinerifampicin-impregnated catheters. This recommendation is based on two RCTs showing that minocycline-rifampicinimpregnated catheters decreased CRBSI [52,53]; however, the guidelines do not mention one meta-analysis with respect to this issue [41]. In that meta-analysis, including these two RCTs [52,53] and another four RCTs, it was found that minocycline-rifampicin-impregnated catheters reduced CRBSI. In addition, the CDC and IDSA guidelines do not mention miconazole-rifampicin-impregnated catheters. However, there are some retrospective studies showing that their use reduced CRBSI $[42,50,51]$. Finally, I think that the use of impregnated catheters should be considered in the following circumstances: channelling of vascular access with increased risk of CRBSI (such as the internal jugular with tracheostomy or femoral access), immunocompromised patients or patients with disorders of skin integrity.

\section{Which technique of catheter insertion}

The advantages of ultrasound-guided central venous catheterization include the identification of the position of the desired vein, the detection of anatomic variants and thrombosis within the vessel and the avoidance of inadvertent arterial puncture during the insertion.

A meta-analysis by Hind et al. [54] was performed to determine the utility of real-time two-dimensional (2-D) and Doppler ultrasound guidance for the insertion of CVCs compared with insertion based solely on anatomic landmarks. It included 18 RCTs and 1646 catheters. In the jugular sub-analysis comparing real-time 2-D ultrasound guidance and landmarks with 7 RCTs and 608 catheters, it was found that real time 2-D was associated with a lower failure rate (relative risk $=0.14,95 \% \mathrm{CI}=0.06-0.33 ; p<0.001$ ) and complications (without detailing what complications) related to catheter placement (relative risk $=0.43,95 \%$ $\mathrm{CI}=0.22-0.87 ; p=0.02$ ). One RCT with 52 subclavian catheters found that real time 2-D was associated with a lower failure rate than landmarks method (relative risk = $0.14,95 \% \mathrm{CI}=0.04-0.57 ; p=0.006)$. In one RCT with 40 femoral catheters, a significantly lower failure rate was not found with real time 2-D ultrasound compared to landmark methods (relative risk $=0.29,95 \%$ CI $=0.07-1.21$; $p=0.09$ ). There are studies comparing real-time 2-D with Doppler ultrasound guidance; however, the authors made an indirect comparison and found that real-time 2-D could be associated with a lower failure rate in subclavian vein procedures than Doppler ultrasound guidance (relative risk = $0.09,95 \% \mathrm{CI}=0.02-0.38 ; p<0.001)$.

After the meta-analysis, other RCTs have been published to determine the utility of real-time 2-D ultrasound guidance for the insertion of CVCs compared with insertion based solely on anatomic landmarks in jugular [55-57] and subclavian sites [58]. In one RCT by Karakitsos et al. [55] with 900 critical care patients, it was found that internal jugular vein cannulation by real-time 2-D ultrasound-guided method $(n=450)$ compared with landmarks method $(n=450)$ showed a higher successful cannulation rate (100 vs. $94.4 \% ; p<0.001)$, lower rate of puncture of the carotid artery ( 1.1 vs. $10.6 \% ; p<0.001)$, haematoma (0.4 vs. $8.4 \% ; p<0.001)$, haemothorax ( 0 vs. $1.7 \% ; p<0.001)$, pneumothorax (0 vs. 2.4\%; $p<0.001)$ and CRBSI (10.4 vs. $16 \% ; p<0.001)$. One RCT by Milling et al. [56] was designed to compare the success rate of 201 internal jugular venous catheterizations: 60 with dynamic ultrasound (guided by real-time 2-D), 72 with static ultrasound (a quick visualization before the procedure) and 69 with anatomic landmarks. The cannulation success rates were 98\%, 82\% and $64 \%$ respectively, i.e. higher with dynamic and static ultrasound guidance than with the anatomic landmarks method; and dynamic ultrasound outperformed static ultrasound, but it may require more training and personnel [56]. In an observational study, Serafimidis et al. [57] compared internal jugular vein catheterization by traditional anatomic landmarks in 204 patients and by real-time 2-D ultrasound-guided method in 347 patients. The 2-D realtime ultrasound-guided technique was associated with lower catheterization failure rate ( 0 vs. $8.8 \%$; $p<0.05)$, lower pneumothorax rate ( 0 vs. $1.0 \% ; p<0.05)$ and lower carotid artery puncture rate $(0.3$ vs. $7.8 \% ; p<0.05)$ [57]. In one RCT by Fragou et al. [58] with 401 subclavian vein cannulation (200 by real-time 2-D ultrasound-guided and 201 by landmarks method), the 2-D ultrasound-guided method showed higher successful cannulation rate (100 vs. $87.5 \% ; p<0.05)$, lower rate of puncture of the subclavian artery (0.5 vs. $5.4 \%$; $p<0.05)$, haematoma ( 1.5 vs. $5.4 \%$; $p<0.05$ ), haemothorax ( 0 vs. $4.4 \%$; $p<0.05$ ), pneumotho$\operatorname{rax}(0$ vs. $4.9 \% ; p<0.05)$, brachial plexus injury ( 0 vs. $2.9 \%$; $p<0.05)$ and phrenic nerve injury ( 0 vs. $1.5 \% ; p<0.05$ ); a limitation was that the effect on the incidence of CRBSI was not recorded.

A meta-analysis by Rabindranath et al. [59], including 7 RCTs with 830 catheters (648 in jugular, 121 in femoral and 61 in subclavian vein sites), was performed to determine the 
utility of real-time 2-D Doppler ultrasound guidance for the insertion of haemodialysis catheters. Ultrasound guidance compared with insertion based solely on anatomic landmarks decreased the risk of catheter placement failure ( 7 studies and 830 catheters; relative risk $=0.12 ; 95 \% \mathrm{CI}=$ $0.04-0.37$ ), arterial punctures ( 6 trials and 785 catheters; relative risk $=0.22 ; 95 \% \mathrm{CI}=0.06-0.81)$ and haematoma formation (4 trials and 323 catheters; relative risk $=0.27 ; 95 \%$ $\mathrm{CI}=0.08-0.88)$. However, there were no significant differences in the rate of pneumothorax and haemothorax.

A meta-analysis by Shiloh et al. [60] was performed to determine the utility of real-time 2-D ultrasound guidance for radial artery catheterization compared with anatomic landmarks. It included 4 RCTs with a total of 311 subjects (152 subjects by landmarks and 159 subjects by ultrasoundguided group) and ultrasound guidance was associated with an improvement in the likelihood of first-attempt success rate $(27 \%$ vs. $43 \%$; relative risk $=1.71 ; 95 \% \mathrm{CI}=1.25-2.32)$. However, data about other complications of radial artery catheterization (such as catheter-related infection and vascular damage) were not reported.

The CDC and IDSA guidelines for the prevention of intravascular catheter-related infections recommended ultrasound guidance for the placement of CVCs (if this technology is available) to reduce the number of cannulation attempts and mechanical complications. Ultrasound guidance should only be used by those fully trained in the technique (Category 1B) [24]. The CDC and IDSA guidelines do not mention the aspect of reducing CRBSI; however, one RCT found that internal jugular vein cannulation by real-time 2-D ultrasound guidance reduced the incidence of CRBSI [55]. I agree with the recommendation that ultrasound guidance should be used to place CVCs.

\section{Conclusions}

In my opinion, central internal jugular with tracheostomy and femoral venous accesses show higher risk of CRBSI and these accesses could be considered as last resort options. Rifampicin-(minocycline or miconazole) and CHSS-impregnated catheters could be considered in vascular accesses with higher risk of CRBSI (such as internal jugular with tracheostomy or femoral access) or patients with higher risk of CRBSI (immunocompromised or with disorders of skin integrity). Ultrasound guidance could help to reduce mechanical complications.

Acknowledgments : This review was supported, in part, by a grant from Instituto de Salud Carlos III (I3SNSINT-11-063) (Madrid, Spain).

Conflict of interest: none.

\section{References}

1. Vincent JL, Bihari DJ, Suter PM, et al (1995) The prevalence of nosocomial infection in Intensive Care Units in Europe. JAMA 274:639-44

2. Lambert ML, Suetens C, Savey A, et al (2011) Clinical outcomes of health-care-associated infections and antimicrobial resistance in patients admitted to European intensive-care units: a cohort study. Lancet Infect Dis 11:30-8

3. Collignon P, Soni N, Pearson I, et al (1988) Sepsis associated with central vein catheters in critically ill patients. Intensive Care Med 14:227-31

4. Kemp L, Burge J, Choban P, et al (1994) The effect of catheter type and site on infection rates in total parenteral nutrition patients. J Parenter Enteral Nutr 18:71-4

5. Goetz AM, Wagener MM, Miller JM, Muder RR (1998) Risk of infection due to central venous catheters: effect of site of placement and catheter type. Infect Control Hosp Epidemiol 19:842-5

6. Merrer J, De Jonghe B, Golliot F, et al (2001) Complications of femoral and subclavian venous catheterization in critically ill patients. JAMA 286:700-7

7. Gowardman JR, Robertson IK, Parkes S, Rickard CM (2008) Influence of insertion site on central venous catheter colonization and bloodstream infection rates. Intensive Care Med 34:1038-45

8. Brun-Buisson C, Abrouk F, Legrand P, et al (1987) Diagnosis of central venous catheter-related sepsis. Critical level of quantitative tip cultures. Arch Intern Med 147:873-7

9. Lorente L, Henry C, Martín MM, et al (2005) Central venous catheter-related infection in a prospective and observational study of 2595 catheters. Crit Care 9:631-5

10. Marik PE, Flemmer M, Harrison W (2012) The risk of catheterrelated bloodstream infection with femoral venous catheters as compared to subclavian and internal jugular venous catheters: a systematic review of the literature and meta-analysis. Crit Care Med 40:2479-85

11. Lorente L, Jimenez A (2012) Central venous catheter site: should we really stop avoiding the femoral vein? Crit Care Med [in press]

12. Parienti JJ, Thirion M, Mégarbane B, et al (2008) Femoral vs jugular venous catheterization and risk of nosocomial events in adults requiring acute renal replacement therapy: a randomized controlled trial. JAMA 299:2413-22

13. Lorente L, Jimenez A, Castedo J, et al (2007) Internal jugular venous catheter-related bacteremia according to central and posterior accesses. Intensive Care Med 33:1071-5

14. Coffin SE, Klompas M, Classen D, et al (2008) Practice recommendation of Society for Healthcare Epidemiology of America/ Infectious Diseases Society of America (SHEA/IDSA). Strategies to prevent ventilator-associated pneumonia in acute care hospitals. Infect Control Hosp Epidemiol 29(Suppl 1):S31-S40

15. Lorente L, Jiménez A, García C, et al (2008) Catheter-related bacteremia from femoral and central internal jugular venous access. Eur J Clin Microbiol Infect Dis 27:867-71

16. Lorente L, Jiménez A, Galván R, et al (2007) Equivalence of posterior internal jugular and subclavian accesses in the incidence of central venous catheter related bacteremia. Intensive Care Med 33:2230-1

17. Michel L, McMichan JC, Bachy JL (1979) Tracheostomy and indwelling central venous line: a hazaradous combination? Intensive Care Med 5:83-6

18. Dusan C, Bouchard P, Goudot B, et al (1981) Bacteriologic study of indwelling central venous catheters. Factors influencing the risk of microbial colonization and sepsis. Anesth Analg (Paris) $38: 347-50$ 
19. Garnacho-Montero J, Aldabó-Pallás T, Palomar-Martínez M, et al (2008) Risk factors and prognosis of catheter-related bloodstream infection in critically ill patients: a multicenter study. Intensive Care Med 34:2185-93

20. Lorente L, Jiménez A, Martín MM, et al (2009) Influence of tracheostomy on the incidence of central venous catheter-related bacteremia. Eur J Clin Microbiol Infect Dis 28:1141-5

21. Lorente L, Jiménez A, Naranjo C, et al (2010) Higher incidence of catheter-related bacteremia in jugular site with tracheostomy than in femoral site. Infect Control Hosp Epidemiol 31:311-3

22. Lorente L, Jiménez A, Martín MM, et al (2011) Lower incidence of catheter-related bloodstream infection in subclavian venous access in the presence of tracheostomy than in femoral venous access: prospective observational study. Clin Microbiol Infect $17: 870-2$

23. Lorente L, Jiménez A, Roca I, et al (2011) Influence of tracheostomy on the incidence of catheter-related bloodstream infection in the catheterization of jugular vein by posterior access. Eur J Clin Microbiol Infect Dis 30:1049-51

24. O'Grady NP, Alexander M, Burns LA, et al (2011) Guidelines for the prevention of intravascular catheter-related infections. Clin Infect Dis 52:e162-e93

25. Scheer B, Perel A, Pfeiffer UJ (2002) Clinical review: complications and risk factors of peripheral arterial catheters used for haemodynamic monitoring in anaesthesia and intensive care medicine. Crit Care 6:199-204

26. Thomas F, Burke JP, Parker J, et al (1983) The risk of infection related to radial vs femoral sites for arterial catheterization. Crit Care Med 11:807-12

27. Singh S, Nelson N, Acosta I, et al (1982) Catheter colonization and bacteremia with pulmonary and arterial catheters. Crit Care Med 10:736-9

28. Traore O, Liotier J, Souweine B (2005) Prospective study of arterial and central venous catheter colonization and of arterial and central venous catheter-related bacteremia in intensive care units. Crit Care Med 33:1276-80

29. Frezza EE, Mezghebe H (1998) Indications and complications of arterial catheter use in surgical or medical intensive care units: analysis of 4932 patients. Am Surg 64:127-31

30. Furfaro S, Gauthier M, Lacroix J, et al (1991) Arterial catheterrelated infections in children. Am J Dis Child 145:1037-42

31. Pinilla JC, Ross DC, Martin T, Crump H (1983) Study of the incidence of intravascular catheter infection and associated septicaemia in critically ill patients. Crit Care Med 11:21-5

32. Koh DB, Gowardman JR, Rickard CM, et al (2008) Prospective study of peripheral arterial catheter infection and comparison with concurrently sited central venous catheters. Crit Care Med 36:397-402

33. Lorente L, Santacreu R, Martín MM, et al (2006) Arterial catheter-related infection of 2,949 catheters. Crit Care 10:R83

34. Lorente L, Jiménez A, Jiménez JJ, et al (2010) Higher arterial catheter-related infection rates in femoral than in dorsalis pedis access. J Hosp Infect 74:365-9

35. Lorente L, Jiménez A, Martín MM, et al (2010) Lower arterial catheter-related infection in brachial than in femoral access. Am J Infect Control 38:e40-e2

36. Lorente L, Jiménez A, Martín MM, et al (2011) Lower catheterrelated bloodstream infection in cubital than in femoral artery access. Scand J Infect Dis 43:814-7

37. Veenstra DL, Saint S, Saha S, et al (1999) Efficacy of antisepticimpregnated central venous catheters in preventing catheterrelated bloodstream infection: a meta-analysis. JAMA 281 (3):261-7

38. Brun-Buisson C, Doyon F, Sollet JP, et al (2004) Prevention of intravascular catheter-related infection with newer chlorhexidine- silver sulfadiazine-coated catheters: a randomized controlled trial. Intensive Care Med 30:837-43

39. Ostendorf T, Meinhold A, Harter C, et al (2005) Chlorhexidine and silver-sulfadiazine coated central venous catheters in haematological patients-a double-blind, randomised, prospective, controlled trial. Support Care Cancer 13:993-1000

40. Rupp ME, Lisco SJ, Lipsett PA, et al (2005) Effect of a secondgeneration venous catheter impregnated with chlorhexidine and silver sulfadiazine on central catheter-related infections: a randomized, controlled trial. Ann Intern Med 143:570-80

41. Falagas ME, Fragoulis K, Bliziotis IA, Chatzinikolaou I (2007) Rifampicin-impregnated central venous catheters: a metaanalysis of randomized controlled trials. J Antimicrob Chemother 59:359-69

42. Lorente L, Lecuona M, Ramos MJ, et al (2008) The use of rifampicin-miconazole-impregnated catheters reduces the incidence of femoral and jugular catheter-related bacteremia. Clin Infect Dis 47:1171-5

43. Darouiche RO, Raad II, Heard SO, et al (1999) A comparison of two antimicrobial-impregnated central venous catheters. Catheter Study Group. N Engl J Med 340:1-8

44. Ramritu P, Halton K (2008) A systematic review comparing the relative effectiveness of antimicrobial-coated catheters in intensive care units. Am J Infect Control 36:104-17

45. Hockenhull JC, Dwan K, Boland A, et al (2008) The clinical effectiveness and cost-effectiveness of central venous catheters treated with anti-infective agents in preventing bloodstream infections: a systematic review and economic evaluation. Health Technol Assess 12:1-154

46. Pai MP, Pendland SL, Danziger LH (2001) Antimicrobialcoated/bonded and -impregnated intravascular catheters. Ann Pharmacother 35:1255-63

47. Saint S, Veenstra DL, Lipsky BA (2000) The clinical and economic consequences of nosocomial central venous catheterrelated infection: are antimicrobial catheters useful? Infect Control Hosp Epidemiol 21:375-80

48. Pittet D, Tarara D, Wenzel RP (1994) Nosocomial bloodstream infection in critically ill patients. Excess length of stay, extra costs, and attributable mortality. JAMA 271:1598-601

49. Dimick JB, Pelz RK, Consunji R, et al (2001) Increased resource use associated with catheter-related bloodstream infection in the surgical intensive care unit. Arch Surg 136:229-34

50. Lorente L, Lecuona M, Ramos MJ, et al (2011) Lower assistance costs using rifampicin-miconazole-impregnated catheters than standard catheters. Am J Infect Control 39:895-7

51. Lorente L, Lecuona M, Ramos MJ, et al (2012) Rifampicinmiconazole-impregnated catheters save cost in jugular venous sites with tracheostomy. Eur J Clin Microbiol Infect Dis 31:1833-6

52. Raad I, Darouiche R, Dupuis J, et al (1997) Central venous catheters coated with minocycline and rifampin for the prevention of catheter-related colonization and bloodstream infections. A randomized, double-blind trial. The Texas Medical Center Catheter Study Group. Ann Intern Med 127:267-74

53. Hanna H, Benjamin R, Chatzinikolaou I, et al (2004) Long-term silicone central venous catheters impregnated with minocycline and rifampin decrease rates of catheter-related bloodstream infection in cancer patients: a prospective randomized clinical trial. $\mathrm{J}$ Clin Oncol 22:3163-71

54. Hind D, Calvert N, McWilliams R, et al (2003) Ultrasonic locating devices for central venous cannulation: meta-analysis. BMJ 327:361

55. Karakitsos D, Labropoulos N, De Groot E, et al (2006) Real-time ultrasound-guided catheterisation of the internal jugular vein: a prospective comparison with the landmark technique in critical care patients. Crit Care 10:R162 
56. Milling TJ Jr, Rose J, Briggs WM, et al (2005) Randomized, controlled clinical trial of point-of-care limited ultrasonography assistance of central venous cannulation: the Third Sonography Outcomes Assessment Program (SOAP-3) Trial. Crit Care Med 33:1764-9

57. Serafimidis K, Sakorafas GH, Konstantoudakis G, et al (2009) Ultrasound-guided catheterization of the internal jugular vein in oncologic patients; comparison with the classical anatomic landmark technique: a prospective study. Int J Surg 7:526-8

58. Fragou M, Gravvanis A, Dimitriou V, et al (2011) Real-time ultrasound-guided subclavian vein cannulation versus the land- mark method in critical care patients: a prospective randomized study. Crit Care Med 39:1607-12

59. Rabindranath KS, Kumar E, Shail R, Vaux E (2011) Use of realtime ultrasound guidance for the placement of hemodialysis catheters: a systematic review and meta-analysis of randomized controlled trials. Am J Kidney Dis 58:964-70

60. Shiloh AL, Savel RH, Paulin LM, Eisen LA (2011) Ultrasoundguided catheterization of the radial artery: a systematic review and meta-analysis of randomized controlled trials. Chest 139:524-9 